\title{
A funcionalidade de idosos institucionalizados: uma revisão integrativa
}

\author{
The functioning of institutionalized elderly: an integrative review
}

El funcionamiento de los ancianos institucionalizados: una revisión integradora

\begin{abstract}
Dhéssy Emelly Travassos Gama ${ }^{1 *}$, Marcella Ayonan dos Santos Silva ${ }^{1}$, Paulo Henrique Ramos Pimentel ${ }^{1}$.
\end{abstract}

\begin{abstract}
RESUMO
Objetivo: Investigar através de uma revisão integrativa da literatura o nível de funcionalidade de idosos institucionalizados. Métodos: Trata-se de uma revisão de literatura do tipo integrativa, para tanto, foi realizado uma busca nas bases de dados Scientific Electronic Library Online (Scielo), Literatura Latino-Americana e do Caribe em Ciências da Saúde (Lilacs) e na National Library of Medice (PubMed). Foram utilizados artigos científicos caracterizados como ensaio clinico publicados nos últimos 5 anos (2017 - 2021) e referidos na língua portuguesa e inglesa que apresentem em seu conteúdo reação direta com o objetivo deste trabalho. Resultados: Foram selecionados 9 artigos. O nível de dependência funcional mostrou-se presente majoritariamente dentre os idosos institucionalizados, principalmente para as Atividades Básicas de Vida Diária e Atividades Instrumentais de Vida Diária, esse número foi mais prevalente em mulheres, sendo que, apenas dois dos artigos utilizados relataram maior grau de funcionalidade nos indivíduos avaliados, além disso, não houve dados significativos relacionados ao nível de funcionalidade entre indivíduos com doenças crônicas não transmissíveis. Considerações finais: Em suma, a institucionalização pode ser um fator que contribuirá para o desenvolvimento de alterações na capacidade funcional dos idosos e assim acarretar maior dependência na realização das atividades de vida diária.
\end{abstract}

Palavras-chave: Instituição de longa permanência para idosos, Classificação internacional de funcionalidade, Incapacidade e saúde, Fisioterapia.

\begin{abstract}
Objective: To investigate, through an integrative literature review, the level of functionality of institutionalized elderly people. Methods: This is an integrative literature review. Therefore, a search was performed in the Scientific Electronic Library Online (Scielo), Latin American and Caribbean Literature in Health Sciences (Lilacs) and National databases. Library of Medicine (PubMed). Scientific articles characterized as clinical trials published in the last 5 years $(2017$ - 2021) and referred to in Portuguese and English that present in their content a direct reaction with the objective of this work were used. Results: 9 articles were selected. The level of functional dependence was mostly present among institutionalized elderly, mainly for Basic Activities of Daily Living and Instrumental Activities of Daily Living, this number was more prevalent in women, and only two of the articles used reported a higher degree of functionality in the individuals evaluated, in addition, there were no significant data related to the level of functionality among individuals with chronic non-communicable diseases. Final considerations: In short, institutionalization can be a factor that will contribute to the development of changes in the functional capacity of the elderly and thus lead to greater dependence in carrying out activities of daily living.
\end{abstract}

Keywords: Long-stay institution for the elderly, International classification of functioning, Disability and health, Physiotherapy.

${ }^{1}$ Centro Universitário da Amazônia (UNAMA), Santarém - PA. *E-mail: gamadh14@gmail.com

SUBMETIDO EM: 10/2021

ACEITO EM: 10/2021

PUBLICADO EM: 10/2021 


\section{RESUMEN}

Objetivo: Investigar, a través de una revisión integradora de la literatura, el nivel de funcionalidad de las personas mayores institucionalizadas. Métodos: Se trata de una revisión integrativa de la literatura, por lo que se realizó una búsqueda en la Biblioteca Electrónica Científica en Línea (Scielo), Literatura Latinoamericana y del Caribe en Ciencias de la Salud (Lilacs) y en bases de datos Nacionales Biblioteca de Medicina (PubMed). Se utilizaron artículos científicos caracterizados como ensayos clínicos publicados en los últimos 5 años (2017 - 2021) y referidos en portugués e inglés que presentan en su contenido una reacción directa con el objetivo de este trabajo. Resultados: Se seleccionaron 9 artículos. El nivel de dependencia funcional estuvo mayoritariamente presente en ancianos institucionalizados, principalmente para Actividades Básicas de la Vida Diaria y Actividades Instrumentales de la Vida Diaria, este número fue más prevalente en mujeres, y solo dos de los artículos utilizados reportaron un mayor grado de funcionalidad en los individuos evaluaron, además, no hubo datos significativos relacionados con el nivel de funcionalidad entre individuos con enfermedades crónicas no transmisibles. Consideraciones finales: En definitiva, la institucionalización puede ser un factor que contribuya al desarrollo de cambios en la capacidad funcional de las personas mayores y, por tanto, conlleve a una mayor dependencia en el desempeño de las actividades de la vida diaria.

Palabras clave: Institución de larga estancia para el adulto mayor, Clasificación internacional del funcionamiento, Discapacidad y salud, Fisioterapia.

\section{INTRODUÇÃO}

Devido ao aumento da população idosa pela redução da taxa de fecundidade, coligado ao déficit funcional e diminuição da disponibilidade de recursos familiares, a procura por Instituições de Longa Permanência para Idosos (ILPIs) vem crescendo cada vez mais. Essas instituições surgiram no Brasil no final do século XIX, são lugares especializados para proporcionar assistência de acordo com as necessidades dos indivíduos com idade mínima de 60 anos sem limite de idade máxima e geralmente aqueles que possuem limitações para realizar suas Atividades de Vida Diária (AVDs). Antigamente os termos utilizados eram: casas de repouso, asilos, abrigos entre outros, porém foi proposto pela Sociedade Brasileira de Geriatria e Gerontologia (SBGG) para ser utilizado "Instituições de Longa Permanência", pois foi notado que os idosos também necessitam de assistência à saúde em geral (FAGUNDES KVDL, et al., 2017; POLTRONIERI BC, et al., 2019).

O levantamento realizado pelo Instituto Brasileiro de Geografia e Estatística (IBGE) relata que $17,3 \%$ dos envelhecimento populacional está acelerado, em 2043 a proporção das pessoas com 60 anos ou mais representará um quarto da população Brasileira, para 2050, segundo as estatísticas, $80 \%$ dessa população viverão em países de baixa e média renda, com tendência a maiores complicações que irão repercutir de forma negativa nos familiares e principalmente nos idosos, assim comprometendo sua qualidade de vida (IBGE, 2018).

O envelhecimento é considerado um processo natural e complexo, que promove alterações biológicas, psicológicas e sociais no indivíduo. Os idosos apresentam características individuais, com o passar do tempo o corpo pode apresentar algumas disfunções como: articulações mais enrijecidas, alterações de equilíbrio, redução de massa e força muscular, entre outros. Portanto, se faz necessário a compreensão desse desenvolvimento, assim como o conhecimento dos fatores que levam as incapacidades funcionais, pois possui efeitos relacionados ao bem-estar e como consequência prejuízos tanto para idosos quanto ao estado (ALMEIDA P, et al., 2017; NETO JO, et al., 2019).

O processo de senescência é definido como resultado de demasiados impactos moleculares e celulares que ocorrem ao decorrer dos anos. Em países desenvolvidos o crescimento da população de terceira idade se dá de forma lenta e em países em desenvolvimento de forma mais rápida. Com o avançar da idade, há o surgimento de diversas alterações como perdas gradativas das funções, de papéis sociais e o agravo de doenças crônicas não transmissíveis e degenerativas, culminando em sequelas limitantes que afetam não apenas o caráter físico, mas também as atividades e a participação social, ocasionando consequências significativas como institucionalização, incapacidade funcional e em última estância a morte (MATOS FS, et al., 2018; ORGANIZAÇÃO PAN-AMERICANA DE SAÚDE (OPAS), 2018). 
A incapacidade funcional é um fator carente de ajuda, definida como a dificuldade em exercer atividades do dia-a-dia devido a problemas de saúde que estão relacionados a fatores multidimensionais, ocasionando maior vulnerabilidade e dependência. Além disso, o déficit funcional acarreta insegurança e aumenta o risco de quedas em idosos, leva a hospitalização e consequentemente gera impactos negativos na mobilidade, assim como eleva a prevalência de estresse na terceira idade em virtude das suas limitações, contribuindo para a diminuição do bem-estar e da qualidade de vida (NUNES JD, et al., 2017; LOPES DGC, et al., 2021).

A população com idade igual ou maior que 60 anos como já dito, vem crescendo a cada década, com isso, caracterizar a qualidade de vida não é simples, pois envolve diversos fatores e torna-se muito amplo. A funcionalidade está diretamente ligada a qualidade de vida do idoso, pois se dá como a capacidade do indivíduo em manter as funções físicas e mentais necessárias para conservar sua autonomia e independência. É vista como um item primordial na atenção à saúde do idoso, uma vez que está expande o campo de visão acerca do processo de avaliação saúde doença. A mesma pode ser avaliada considerando dois aspectos: Atividades Instrumentais de Vida Diária (AIVD) e Atividades Básicas de Vida Diária (ABVD) (TALARSKA D, et al., 2017; CARVALHO TC, et al., 2018).

As AIVD's correspondem às ações mais complexas que referem-se a competência de ter independência na sociedade como: fazer compras, usar o telefone, dirigir, usar transporte coletivo, manusear o próprio dinheiro ou administrar seus remédios. Já as ABVD's são aquelas referentes ao autocuidado, como banharse, vestir-se, alimentar-se, caminhar da sala para a cozinha ou transferir-se da cama para a cadeira. Dessa forma, a avalição da capacidade funcional tornou-se fundamental para a elaboração das condutas terapêuticas, visando a individualidade e assim possibilitando o melhor acompanhamento da situação clínica funcional do idoso (LEAL RC, et al., 2020).

O maior comprometimento funcional dos idosos está relacionado às AIVDs, devido à alta complexidade que recruta maior desempenho físico e cognitivo quando comparada às ABDVs. Vale ressaltar, que o sexo feminino está mais propenso a dependência funcional, visto que, possuem maior expectativa de vida e vulnerabilidade as doenças crônicas, além disso, pode-se considerar também outros fatores de risco que contribuem para o déficit funcional em ambos os sexos como, maior idade e o baixo nível de atividade física. Desse modo, a avaliação da capacidade funcional da pessoa idosa é de suma importância, uma vez que está fornece dados fundamentais para a assistência multiprofissional, que pode intervir com o intuito de minimizar ou evitar a perda da capacidade funcional e prevenir complicações (PARISI DP, et al., 2019; NETO JO, et al., 2019).

Toda via, de acordo com Gomes CS, et al. (2017) faltam instrumentos de avaliação da pessoa idosa institucionalizada que contemplem os itens propostos pela Classificação Internacional de Funcionalidade, Incapacidade e Saúde (CIF), respeitando seu modelo biopsicossocial, uma vez que grande parte das escalas abrangem apenas o domínio de atividades, negligenciando os fatores ambientais, contextuais, funções e estruturas corporais.

A CIF é uma ferramenta utilizada para classificar e descrever as condições funcionais que podem ou não estar associadas a doenças, assim como para identificar os fatores ambientais e pessoais que interferem de forma positiva ou negativa ás condições de saúde, faz parte da família de classificações de referência da Organização Mundial da Saúde (OMS) juntamente com a CID (Classificação estatística de doenças e problemas relacionados a saúde). Ela abrange várias dimensões da saúde como, biológicas, patológicas, ambientais, culturais e sociais, por isso possui também uma interação multidimensional. A mesma define a funcionalidade como um termo que abrange todas as funções do corpo, atividades e participação de forma semelhante, e a incapacidade como uma condição que engloba deficiências, limitações de atividades ou restrição na participação social, ambas relacionadas ao meio ambiente em que a pessoa vive (ANDRADE LEL, et al., 2017; CARRASCOSA CA e RIBEIRO IL, 2018).

De acordo com Ribeiro YHCP e Romão JFF (2017), as escalas e testes mais utilizadas para avalição do idoso são: Medida de Independência Funcional (MIF), Índice de Katz que são aplicadas para análise da Atividades Básicas de Vida Diária, escala de Lawton-Brody para graduar o desempenho durante realização das Atividades Instrumentais de Vida Diária, teste Timed Up and Go (TUG) que está relacionado as medidas de desempenho e para avaliar as funções cognitivas pode-se citar o Mini Exame do Estado Mental (MEEM). 
A MIF é um instrumento que avalia o nível de incapacidade funcional de pacientes com restrições à nível motor e cognitivo/social relacionados ao autocuidado: alimentação, higiene pessoal, banho, vestir metade superior e inferior do corpo, uso do vaso sanitário, controle esfincteriano, controle miccional, transferências de leito, cadeira, cadeira de rodas, transferência: para vaso sanitário, transferências para banheira ou chuveiro, locomoção: em escadas, compreensão, expressão, interação social, resolução de problemas e memória. Devido a sua confiabilidade e praticidade a MIF tem sido uma das principais escalas utilizadas no meio cientifico para realização de estudos transversais, assim como na atenção à saúde do idoso principalmente no âmbito ambulatorial, onde auxilia na construção do plano terapêutico como: metas e objetivos através de seus resultados, que possibilitam também a modificação dessa abordagem conforme a evolução do paciente baseado no aumento dos Scores (RIBEIRO DKMN, et al., 2017; JESUS MBC, et al., 2017).

O Índice de Katz, é uma escala utilizada para entender a capacidade funcional do indivíduo, contendo a realização de funções básicas como: banhar-se, vestir-se, transferência, continência, alimentação e utilizar o banheiro. A classificação se dá a partir dos resultados, pode ser definida como: independente, dependente parcial ou dependente total. Ela leva em conta a independência e a autonomia das pessoas, sendo relacionadas à capacidade de efetuar suas atividades básicas da vida diária. Sendo capaz de definir os cuidados essenciais e evitar agravos que possam ser causados por uma dependência presente, visando aperfeiçoar a qualidade de vida e trazer maior independência do idoso (MENDES SO, et al., 2020; LEITE AK, et al., 2020).

Já o MEEM é um dos testes mais utilizados no mundo para avalição da função cognitiva, além de auxiliar no processo de diagnóstico de demência. Esse teste explora memorias de curto prazo e evocação, orientação de tempo e espaço, cálculos, atenção, assim como habilidades visuais e/ou espaciais e de linguagem, possui Score máximo de 30 pontos, onde 24 pontos para indivíduos com nível superior refere-se a possível demência, menor que 18 pontos para indivíduos com fundamental e menor que 14 pontos para os não alfabetizados também é preditivo para demência (RIBEIRO YHCP e ROMÃO JFF, 2017; MELO M e CADER AS, 2018).

O TUG é um dos instrumentos de avaliação da mobilidade e de equilíbrio funcional mais utilizados a nível nacional e internacional. É um teste rápido e prático em virtude da sua simplicidade, pois não requer nenhum equipamento especial. Tem a finalidade de analisar clinicamente mudanças do equilíbrio dinâmico em pessoas idosas durante a execução de atividades com grandes riscos de queda. Este teste calcula em segundos o tempo que um sujeito leva para levantar de uma cadeira, andar uma distância de 3 metros, virar, retornar para a cadeira e sentar novamente. O mesmo tem sido bastante empregado na prática clínica como indicador de resultado para avaliar o equilíbrio dinâmico, capacidade funcional ou o risco de quedas em adultos e principalmente idosos. Dos variados testes que podem ser usados para avaliar a funcionalidade, ele é um dos mais utilizados com a população idosa (FILIPPIN LI, et al., 2017; ROSA AMP, et al., 2017).

Diante disso, o presente estudo teve como objetivo analisar por meio de uma revisão integrativa da literatura a funcionalidade de idosos institucionalizados.

\section{MÉTODOS}

O estudo atual é caracterizado como uma revisão de literatura do tipo integrativa, para tanto, foi realizado uma busca nas bases de dados Scientific Electronic Library Online (Scielo), Literatura Latino-Americana e do Caribe em Ciências da Saúde (Lilacs) e National Library of Medice (PubMed) com os termos: "Instituição de Longa Permanência para Idosos", "Classificação Internacional de Funcionalidade, Incapacidade e Saúde", "Fisioterapia" e seus correspondentes na língua inglesa: "Long-stay Institution for the Elderly"; "International Classification of Functioning","Disability and Health"; "Physiotherapy".

Como critérios de inclusão foram utilizados artigos científicos caracterizados como ensaio clinico publicados nos últimos 5 anos (2017 - 2021) e redigidos na língua portuguesa e inglesa que apresentem em seu conteúdo reação direta com o objetivo deste trabalho. Foram excluídos artigos de revisão de literatura, estudos de casos, estudos experimentais com modelo animal, projetos de pesquisa em andamento, ou que não se enquadrarem aos critérios de inclusão. 
A metodologia foi dividida em 4 etapas, sendo elas: 1르 Etapa - Aquisição de material para estudo, foram realizadas pesquisas nas seguintes bases de dados: Scielo, Lilacs e PubMed, utilizando os seguintes descritores: Instituição de Longa Permanência para Idosos; Classificação Internacional de Funcionalidade, Incapacidade e Saúde; Fisioterapia e seus correspondentes na língua inglesa: "Long-stay Institution for the Elderly"; "International Classification of Functioning", "Disability and Health"; "Physiotherapy". Finalizando essa primeira etapa com a seleção dos estudos que fizeram parte da revisão seguindo os critérios de inclusão e de exclusão (Figura 1).

Já a $2^{\underline{a}}$ etapa refere-se à organização dos dados, onde foi dividida em 2 momentos, a) Leitura exploratória de todo material selecionado; b) Registro e organização das informações extraídas das fontes em instrumentos específicos (Quadro 1). Seguindo a sequência, título, autores/ano, amostra/idade, metodologia e resultados.

$\mathrm{Na} 3^{\text {a }}$ etapa, temos análise e interpretação dos dados, nesta etapa foram realizadas leituras analíticas com a finalidade de ordenar e sumariar as informações contidas nas fontes, de forma que estas possibilitaram a obtenção de respostas ao problema da pesquisa.

E por últimos a $4^{\mathrm{a}}$ Etapa que se refere a construção da revisão que foi construído o estudo, produto da análise dos dados para obtenção da resposta da questão problema deste projeto.

Por ter como referência bases públicas, não foi necessária a submissão ao Comitê de Ética e Pesquisa, respeitando, porém, os preceitos éticos estabelecidos na resolução número 466/2012 do Conselho Nacional de Saúde.

Figura 1 - Fluxograma da pesquisa.

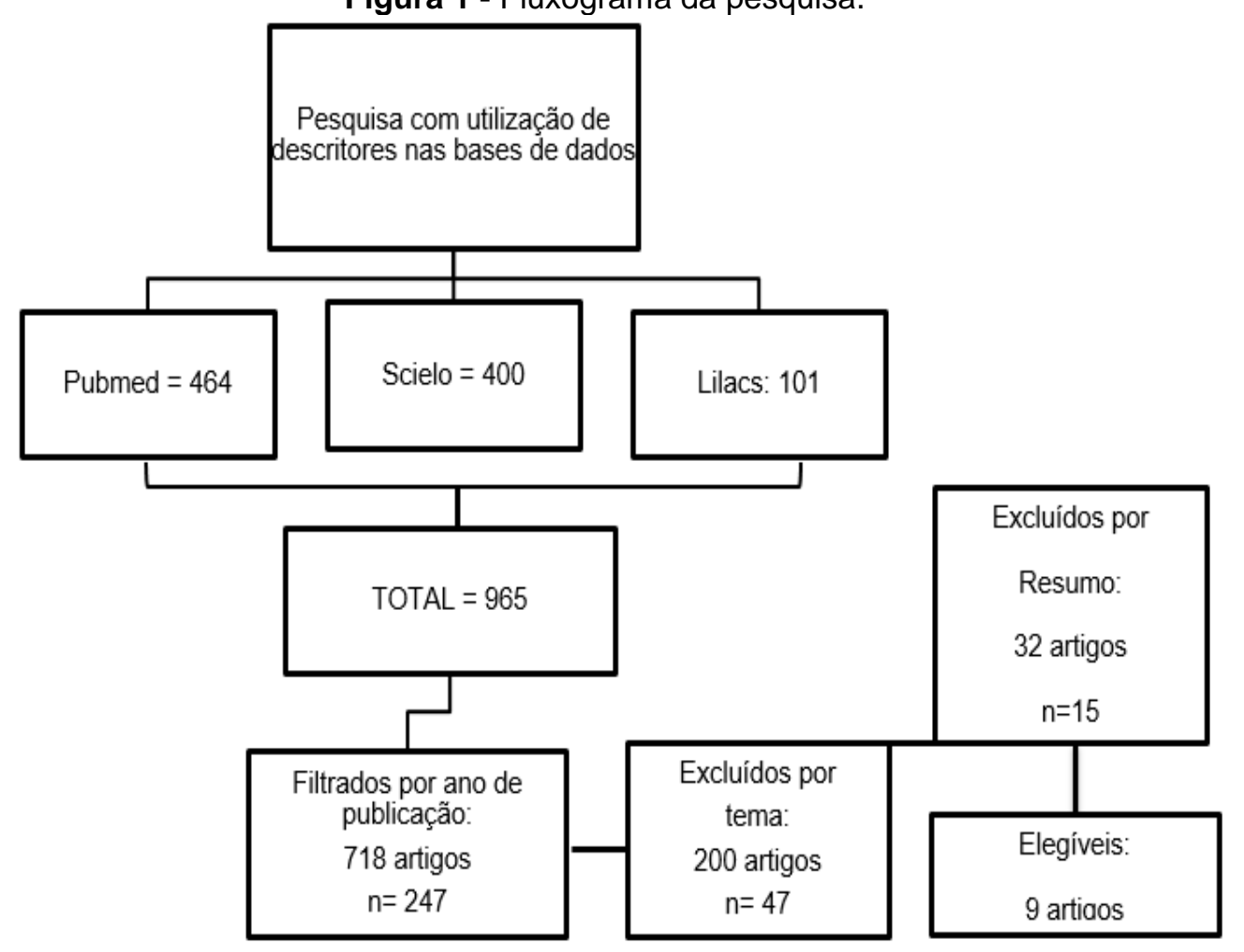

Fonte: Gama DET, et al., 2021.

\section{RESULTADOS}

Foram encontrados 965 artigos nas bases de dados, após aplicação dos critérios de inclusão, exclusão e leitura completa dos artigos filtrados, foram selecionados 9 artigos para compor a presente revisão integrativa acerca da funcionalidade de idosos institucionalizados. As características dos estudos encontrados podem ser observadas no Quadro 1. 
Quadro 1: Síntese dos principais achados sobre a funcionalidade de idosos institucionalizados.

\begin{tabular}{|c|c|c|c|c|c|}
\hline № & Título & Autores (ano) & Amostra/ Idade & Metodologia & Resultados \\
\hline 1 & $\begin{array}{l}\text { Avaliação da funcionalidade de idosos } \\
\text { institucionalizados: relação entre a MIF e a } \\
\text { ICF. }\end{array}$ & $\begin{array}{l}\text { Fleig TCM e } \\
\text { Oliveira MR } \\
(2017)\end{array}$ & $\begin{array}{l}55 \text { pessoas ( } 15 \mathrm{M} \text { e } 40 \mathrm{~F}) \text {, } \\
\text { com idade igual ou superior a } \\
60 \text { anos. }\end{array}$ & $\begin{array}{l}\text { Formulário sociodemográfico, } \\
\text { MIF e CIF. }\end{array}$ & $\begin{array}{l}\text { Foi observado uma maior independência completa de } \\
47,3 \% \text { e dependência mínima de } 23,6 \% \text {, mostrando pouco } \\
\text { comprometimento funcional da populacão. }\end{array}$ \\
\hline 2 & $\begin{array}{l}\text { Análise comparativa da capacidade } \\
\text { funcional de idosos institucionalizados e } \\
\text { não institucionalizados }\end{array}$ & $\begin{array}{l}\text { Leão RO, et al. } \\
\qquad(2017)\end{array}$ & $\begin{array}{l}20 \text { indivíduos divididos em } 2 \\
\text { grupos: ICO: } 10 \text { ( } 5 \mathrm{M} \text { e } 5 \mathrm{~F}) \text { e } \\
\text { IIN: } 10 \text { ( } 4 \mathrm{M} \text { e } 6 \mathrm{~F}) \text {. Idade de } \\
60 \text { a } 80 \text { anos. }\end{array}$ & $\begin{array}{l}\text { MEEM, índice de Katz, Escala de } \\
\text { Lawton e Brody, e o TUG. }\end{array}$ & $\begin{array}{l}\text { Notou-se que houve redução da capacidade funcional dos } \\
\text { idosos institucionalizados quando comparada com não } \\
\text { institucionalizados. }\end{array}$ \\
\hline 3 & $\begin{array}{l}\text { Análise do grau de autonomia de residentes } \\
\text { em uma Instituição de Longa Permanência. }\end{array}$ & $\begin{array}{l}\text { Reis LAM, et al. } \\
\quad(2017)\end{array}$ & $\begin{array}{l}\text { Estudo realizado com } 22 \\
\text { idosos de ambos os sexos, } \\
\text { faixa etária de } 59 \text { a } 75 \text { anos. }\end{array}$ & $\begin{array}{l}\text { Foi utilizado questionário } \\
\text { sociodemográfico Escala de } \\
\text { Katze Escala Lawton \& Brody. }\end{array}$ & $\begin{array}{l}\text { Verificou-se através da escala de Katz que } 85 \% \text { da } \\
\text { amostra apresentou independência para realização das } \\
\text { ABVD e a escala de Lawton-Brody mostrou que } 81,80 \% \\
\text { da amostra apresenta dependência moderada para AIVD. }\end{array}$ \\
\hline 4 & $\begin{array}{l}\text { Capacidade funcional de idosos em } \\
\text { instituição de longa permanência. }\end{array}$ & $\begin{array}{l}\text { Silva CS, et al. } \\
\qquad(2017)\end{array}$ & $\begin{array}{l}10 \text { participantes ( } 3 \mathrm{M} \mathrm{e} 7 \mathrm{~F} \text { ), } \\
\text { com idade média de } 75,2 \pm \\
10,29 \text { anos. }\end{array}$ & $\begin{array}{l}\text { Questionário sociodemográfico e } \\
\text { Escala de Katz. }\end{array}$ & $\begin{array}{l}\text { Observou-se que a maioria da amostra apresentam } \\
\text { dependência para realizar AVD's. }\end{array}$ \\
\hline 5 & $\begin{array}{l}\text { Capacidade funcional de idosos residentes } \\
\text { em uma instituição de longa permanência. }\end{array}$ & $\begin{array}{l}\text { Souza LHR, et } \\
\text { al. (2017) }\end{array}$ & $\begin{array}{l}\text { A amostra constituída por } 32 \\
\text { idosos com idade igual ou } \\
\text { superior a } 60 \text { anos. }\end{array}$ & $\begin{array}{l}\text { Foi utilizado questionário } \\
\text { sociodemográfico Escala de } \\
\text { Katze Escala Lawton \& Brody. }\end{array}$ & $\begin{array}{l}\text { Constatou-se que } 51,3 \% \text { da amostra apresentou } \\
\text { semidependência para AVD e } 65,6 \% \text { para AIVD, além } \\
\text { disso, não houve associação entre o grau de dependência } \\
\text { funcional dos idosos com o sexo. }\end{array}$ \\
\hline 6 & $\begin{array}{l}\text { Avaliação da funcionalidade de idosos } \\
\text { institucionalizados: relação entre o Índice } \\
\text { de Barthel e a Classificação Internacional } \\
\text { de Funcionalidade, } \\
\text { Incapacidade e Saúde (ICF). }\end{array}$ & $\begin{array}{l}\text { Oliveira MR e } \\
\text { Fleig TCM } \\
\text { (2017) }\end{array}$ & $\begin{array}{l}55 \text { idosos ( } 15 \mathrm{M} \mathrm{e} 40 \mathrm{~F}) \text {, com } \\
\text { idade igual ou superior a } 60 \\
\text { anos. }\end{array}$ & $\begin{array}{l}\text { Questionário sociodemográfico, } \\
\text { MEEM, índice de Barthel, CIF. }\end{array}$ & $\begin{array}{l}\text { Foi constatado que a maioria da população apresentava } \\
\text { dependência para realizar suas AVD'S. }\end{array}$ \\
\hline 7 & $\begin{array}{l}\text { Fatores associados à dependência de } \\
\text { idosos residentes em instituições públicas }\end{array}$ & $\begin{array}{l}\text { Scherrer G, et } \\
\text { al. (2019) }\end{array}$ & $\begin{array}{l}154 \text { participantes de ambos } \\
\text { os sexos, com idade igual ou } \\
\text { superior a } 60 \text { anos. }\end{array}$ & $\begin{array}{l}\text { Foi aplicado o MEEM, Indice de } \\
\text { Katz e MNA-SF }\end{array}$ & $\begin{array}{l}\text { De acordo com o índice de Katz, } 57,1 \% \text { da amostra } \\
\text { apresentou independência para realização das ABVD. }\end{array}$ \\
\hline 8 & $\begin{array}{l}\text { Capacidade funcional do idoso } \\
\text { institucionalizado } \\
\text { avaliado pelo KATZ. }\end{array}$ & $\begin{array}{l}\text { Leite AK, et al. } \\
\qquad(2020)\end{array}$ & $\begin{array}{l}38 \text { participantes, ambos os } \\
\text { sexos, com idade média de } \\
74 \text { anos. }\end{array}$ & $\begin{array}{l}\text { Questionário sociodemográfico } \\
\text { escala de Katz. }\end{array}$ & $\begin{array}{l}\text { Verificou-se que } 39,48 \% \text { da amostra apresentou } \\
\text { dependência parcial para atividade de vestir-se, no } \\
\text { entanto, a maioria obteve nível independente para } \\
\text { atividade de transferência. }\end{array}$ \\
\hline 9 & $\begin{array}{l}\text { Avaliação da capacidade funcional dos } \\
\text { idosos residentes em uma instituição de } \\
\text { longa permanência. }\end{array}$ & $\begin{array}{l}\text { Dias FSS, et al. } \\
\text { (2021) }\end{array}$ & $\begin{array}{l}24 \text { indivíduos com } 60 \text { anos de } \\
\text { idade ou mais, sendo } 13 \mathrm{M} \mathrm{e} \\
11 \mathrm{~F} \text {. A idade variou entre } 60 \\
\text { e } 95 \text { anos. }\end{array}$ & $\begin{array}{l}\text { Foi utilizado questionário } \\
\text { sociodemográfico e escala de } \\
\text { Katz. }\end{array}$ & $\begin{array}{l}\text { Observou-se que a amostra apresentou maior índice de } \\
\text { independência para as tarefas de: banhar-se, ir ao } \\
\text { banheiro, transferir-se, continência urinária, fecal e } \\
\text { alimentar-se. }\end{array}$ \\
\hline
\end{tabular}

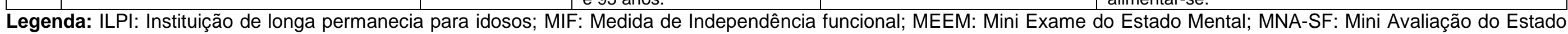

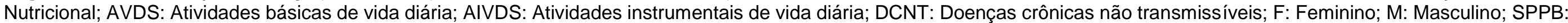

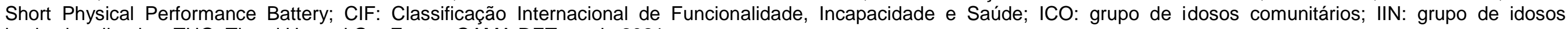
institucionalizados; TUG: Timed Up and Go. Fonte: GAMA DET, et al., 2021. 


\section{DISCUSSÃO}

O processo de senescência é considerado um evento complexo e inevitável caracterizado por alterações em todos os sistemas do corpo, sendo eles: Musculoesquelético, Circulatório, Sensorial, Sistema Nervoso, Endócrino, Urinário, Digestório e dentre outros, que vão influenciar na capacidade de adaptação ao ambiente, acarretando maior predisposição ao indivíduo em desenvolver doenças crônicas degenerativas, déficit funcional para execução de AVD, tornando-o dependente do auxílio de outras pessoas para realizar atividades simples do dia-a-dia como: andar, alimentar-se e vestir-se. Sendo que, a incapacidade é um dos fatores que levam ao aumento na taxa de encaminhamento de idosos às ILPIs (FIGUEREDO FFO, et al., 2019; COSTA RAGC, et al., 2018; ANTONELLI G, et al., 2017).

Reis CCA, et al. (2019) define as ILPIs como organizações designadas ao amparo coletivo a longo prazo de idosos que necessitam, seja por fatores relacionados a fragilidade, ausência de assistência familiar, social e/ou condições financeiras desfavoráveis. Estas, buscam focalizar na independência, autoestima e preservar a individualidade da pessoa idosa, no entanto, na prática, observa-se o decréscimo da autonomia e independência dos mesmos (LOPES VM, et al., 2018). Assim observa Leão RO, et al. (2017) em seu estudo, onde comparou a capacidade funcional de idosos institucionalizados e idosos não institucionalizados e notou a redução significativa da capacidade funcional dos idosos institucionalizados para as AIVDs quando comparado aos não institucionalizados, reforçando os achados de Leite AK, et al. (2020) onde verificou-se que $39,48 \%$ da amostra necessitava de suporte para vestir-se, no entanto, quanto a tarefa de transferência os idosos mostraram maior nível de independência.

A funcionalidade se refere à capacidade de desempenhar AVBD e AIVD, possibilita o cuidar de si mesmo e executar tarefas sem necessidade de ajuda. É um termo que abrange todas as funções do corpo, atividades e participação, inclui a idade avançada, comprometimento cognitivo, histórico de quedas, comorbidade e entre outros (SILVA CS, et al., 2017). Para avaliação da mesma pode-se considerar os seguintes parâmetros: equilíbrio, mobilidade, função cognitiva dentre outros a fim de identificar, tratar e/ou prevenir possíveis agravos, para isso, pode-se utilizar escalas, testes e/ou questionários como a escala de Katz que avalia atividades básicas como: banhar-se, vestir-se, transferência, higiene pessoal, continência e alimentar-se e a escala de Lawton e Brody, utilizada na avaliação da capacidade instrumental de vida diária (ARAÚJO EAT, et al., 2020; DIAS FSS, et al., 2021).

Dentre os estudos analisados, notou-se que grande parte das avaliações foram feitas através da utilização da escala de Katz, sendo que, apenas três estudos optaram por outros instrumentos como a: MIF, CIF, MEEM, Mini Avaliação do Estado Nutricional (MNA-SF), Índice de Barthel e TUG além dos questionários sociodemográfico (FLEIG TCM e OLIVEIRA MR, 2017; LEÃO RO, et al., 2017; REIS LAM, et al., 2017; SILVA CS, et al., 2017; SOUZA LHR, et al., 2017; OLIVEIRA MR e FLEIG TCM, 2017; SCHERRER G, et al., 2019; LEITE AK, et al., 2020; DIAS FSS, et al., 2021).

Fleig TCM e Oliveira MR (2017), observaram através da Medida de Independência Funcional (MIF) que 47,3\% da amostra apresentou independência completa para AVDs e 23,6\% mostraram dependência mínima, em conformidade com os achados de Reis LAM, et al., (2017) que constatou através da escala de Katz, que 85\% da amostra manifestou independência para realização das ABVD, divergindo da escala de Lawton $e$ Brody que classificou $81,80 \%$ dos idosos com dependência moderada para AIDV.

Para Figueiredo FFO, et al. (2019) esse quadro se dá devido ao excesso de ajuda e pouca estimulação que os idosos recebem dos cuidadores nas ILPIs, principalmente aqueles que apresentam maior dificuldade na execução das AVDs, ainda que os mesmos possuam condições de realiza-las. Consoante ao estudo de Oliveira MR e Fleig TCM (2017), onde foi indicado um percentual maior dos participantes com dependência severa, ou seja, baixa capacidade para realizar suas AVD's, corroborando com os achados de Souza LHR, et al., (2017) no qual 51,3\% da amostra apresentou semidependência para AVD e 65,6 \% para AIVD, além disso, não houve associação entre o grau de dependência funcional dos idosos com o sexo.

Verificou-se nos estudos aqui abordados o predomínio de mulheres institucionalizadas, o que pode estar relacionado com os dados da Pesquisa Nacional por Amostras de Domicilio Continua (PNAD) que mostra a prevalência de 51,8\% de mulheres na população Brasileira, o que diverge dos dados de Dias FSS, et al. 
(2021) onde $54,2 \%$ da amostra estudada era do sexo masculino, sendo identificado maior dependência no sexo feminino, principalmente na tarefa de vestir-se (IBGE, 2021). Silva CS, et al. (2017) observou $60 \%$ de dependência em sua amostra, demostrando que quanto maior o tempo de institucionalização maior será a diminuição da capacidade funcional, não só pelo processo de envelhecimento, mas também por aspectos psicológicos.

Ao longo do ciclo da vida ocorrem diversas alterações fisiológicas que se manifestam mais rapidamente na terceira idade, assim como alterações patológicas denominadas, processo de Senilidade, que podem surgir na fase adulta e evoluir com o avançar da idade, em sua maioria é considerada como Doenças Crônicas Degenerativas Não Transmissíveis (DCNT), podendo ocasionar maior grau de dependência do idoso, no que se refere ao desempenho das AVDs e por conseguinte influenciar a sua qualidade de vida. Com isso, tornase oportuno salientar que as DCNTs em idosos podem estar associadas ao declínio funcional que se refere as deficiências, limitações de atividades ou restrição na participação na comunidade (SOUSA HJS, et al., 2019; FIGUEIREDO AEB, et al., 2021).

Ferrarez ML, et al. (2020) analisou em seu estudo que as principais comorbidade presentes são: Hipertensão Arterial Sistêmica (HAS), que pode ser um fator considerável para a redução da capacidade funcional para as AIVDs, seguido de diabetes e depressão, visto que, para Figueiredo AEB, et al. (2021) as DCNTs que apresentam maior prevalência são as cardiocirculatórias, posteriormente a Diabetes Mellitus (DM), Neoplasias e Doenças Respiratórias.

Em contraste ao estudo de Scherrer G, et al. (2019), que propôs comparar o grau de dependência aos fatores sociodemográfico de idosos residentes em instituições públicas, nesse estudo a amostra apresentou média de 2,5 de doenças crônicas, no entanto, esse número não foi considerado significativo para relacionarse ao nível de dependência, além disso $57,1 \%$ dos participantes apresentaram independência para realização das ABVDs.

\section{CONSIDERAÇÕES FINAIS}

Em síntese, verificou-se que a institucionalização pode ser um fator que contribuirá para o desenvolvimento de alterações na capacidade funcional dos idosos e assim acarretar maior dependência na realização das Atividades de Vida Diária. Vale ressaltar, que o processo de senescência e suas variações possuem grande relação com o déficit funcional, portanto, consoante aos estudos nota-se que o declínio funcional está atrelado também a outros fatores e quando associados as Instituições de Longa Permanência podem ser potencializados. Com isso, sugere-se mais estudos acerca da temática, que possam corroborar com a discussão do tema e dispor de resultados que objetive contribuir com o cuidado da pessoa idosa residente de instituições de longa permanência, assim como abordagens que priorizem a funcionalidade.

\section{REFERÊNCIAS}

1. ALMEIDA $P$, et al. Funcionalidade e fatores associados em idosos participantes de grupo de convivência. Revista da Associação Brasileira de Atividade Motora Adaptada, 2017; 18(1): 53-64.

2. ANDRADE LEL, et al. Avaliação do nível de conhecimento e aplicabilidade da Classificação Internacional de Funcionalidade, Incapacidade e Saúde. Saúde Debate, 2017; 41(114): 812-823.

3. ANTONELLI G, et al. Avaliação da capacidade funcional de idosos em uma instituição de longa permanência de Guarapuava- Paraná. Cinergis, 2017; 18(3), 160-163.

4. ARAUJO EAT, et al. A utilização do Îndice de Barthel em idosos brasileiros: uma revisão de literatura. Revista Kairós: Gerontologia, 2020; 23(2), 217-231.

5. CARRASCOSA CA, RIBEIRO IL. Aplicação da Classificação Internacional de Funcionalidade, Incapacidade e Saúde (CIF) em saúde do trabalhador. Revista Brasileira Multidisciplinar, 2018; 21(2).

6. CARVALHO TC, et al. Impacto da hospitalização na funcionalidade de idosos: estudo de coorte. Rev. Bras. Geriatr. Gerontol, 2018; 21(2): 136-144.

7. COSTA RAGC, et al. Assistência em enfermagem ao idoso: Uma Perspectiva da Área de Geriatria e Gerontologia. 2018.

8. DIAS FSS, et al. Avaliação da capacidade funcional dos idosos residentes em uma instituição de longa permanência. Revista Eletrônica Acervo Saúde, 2021; 13(2), 6361-6361.

9. FAGUNDES KVDL, et al. Instituições de longa permanência como alternativa no acolhimento das pessoas idosas. Rev. Salud Pública, 2017; 19 (2): 210-214. 
10. FIGUEIREDO FFO, et al. Idosos que vivem em instituição de longa permanência: vínculo, cuidado da família e capacidade funcional. Dissertação (Mestrado em Família na Sociedade Contemporânea), Universidade Católica do Salvador, 2019.

11. FIGUEIREDO EAB, et al. Doenças crônicas não transmissíveis e suas implicações na vida de idosos dependentes. Ciênc. Saúde Colet. 2021; 26 (01).

12. FILIPPIN LI, et al. Timed Up and Go test no rastreamento da sarcopenia em idosos residentes na comunidade. Rev. Bras. Geriatr. Gerontol, 2017; 20(4): 561-566.

13. FLEIG TCM, OLIVEIRA MR. Avaliação da funcionalidade de idosos institucionalizados: relação entre a MIF e a ICF. Cinergis, 2017; 18(3): 190-195.

14. GOMES CS, et al. Assessment instruments of functioning in Brazilian elderly and the ICF: a systematic review. Fisioter Mov, 2017; 30(3): 625-37.

15. INSTITUTO BRASILEIRO DE GEOGRAFIA E ESTATÍSTICA (IBGE). Conheça o Brasil - População quantidade de homens e mulheres: IBGE, 2021. Disponível em: https://educa.ibge.gov.br/ jovens/conheca-o-brasil/populacao/18320quantidade-de-homens-e-mulheres.html. Acessado em: 15 de Outubro de 2021.

16. JESUS MBC, et al. O impacto de atividades coletivas na capacidade funcional em idosos. Rev. Saúde.Com 2017; 13(2): 894-901.

17. LEAL RC, et al. Efeitos do envelhecer: grau de dependência de idosos para as atividades da vida diária. Brazilian Journal of Development, 2020; 6(7): 53931-53940.

18. LEÃO RO, et al. Análise comparativa da capacidade funcional de idosos institucionalizados e não institucionalizados. FisiSenectus, 2017; 5(1): 3-12.

19. LEITE AK, et al. Capacidade funcional do idoso institucionalizado avaliado pelo KATZ. Revista enfermagem atual in derme, 2020; 90(21): 101-109.

20. LOPES DGC, et al. Treinamento com realidade virtual não imersiva é efetivo na melhora da funcionalidade de idosos institucionalizados e uma opção de atividade física segura nos momentos de restrição: um estudo piloto. Acta Fisiatr, $2021 ; 28(2): 86-91$.

21. LOPES VM, et al. What taken the elderly people to institutionalization? Rev Enferm UFPE on line, 2018;12(9):242835.

22. MATOS FS, et al. Redução da capacidade funcional de idosos residentes em comunidade: estudo longitudinal. Ciência \& Saúde Coletiva, 2018; 23(10): 3393-3401.

23. MELO M, CADER AS. Caracterização e correlação do estado mental e da capacidade funcional de idosos asilados com mal de Alzheimer no brasil e Paraguai. Revista Brasileira de Neurologia e Psiquiatria, 2018; 22(2): 110-123.

24. MENDES SO, et al. Validade e confiabilidade da Escala Índice de Katz Adaptada. Research, Society and Development, 2020; 9(4).

25. NETO JO, et al. Avaliação da funcionalidade em idosas institucionalizadas. Revista Desafios, 2019; 6(03).

26. NUNES JD, et al. Indicadores de incapacidade funcional e fatores associados em idosos: estudo de base populacional em Bagé, Rio Grande do Sul. Epidemiol. Serv. Saude, 2017; 26(2): 295-304.

27. OLIVEIRA MR, FLEIG TCM. Avaliação da funcionalidade de idosos institucionalizados: relação entre o Índice de Barthel e a Classificação Internacional de Funcionalidade, Incapacidade e Saúde (ICF). Revista Brasileira de Iniciação Científica, 2017; 4(9).

28. ORGANIZAÇÃO PAN-AMERICANA DE SAÚDE (OPAS). Envelhecimento e saúde. Brasília, 2018. Disponível em: https://www.paho.org/pt/envelhecimento-saudavel. Acessado em: 15 de Outubro de 2021.

29. POLTRONIERI BC, et al. Violência no cuidado em Instituições de Longa Permanência para idosos no Rio de Janeiro: Percepções de gestores e profissionais. Saúde soc, 2019; 28(2): 215-226.

30. PARISI DP, et al. Perfil sociodemográfico, clínico, de avaliação cognitiva e funcional de idosas institucionalizadas em ILPI's. Rev. Longeviver, 2019; 1(4)

31. REIS CCA, et al. Ser Pessoa Idosa Institucionalizada: Sentido do Vivido à Luz da Fenomenologia Heideggeriana. Rev Bras Enferm, 2019;72(6):1710-6.

32. REIS LAM, et al. Análise do grau de autonomia de residentes em uma Instituição de Longa Permanência. Revista Kairós-Gerontologia, 2017; 20(23), 191-204.

33. RIBEIRO DKMN, et al. O emprego da medida de independência funcional em idosos. Rev Gaúcha Enferm, 2017; $38(4)$.

34. RIBEIRO YHCP, ROMÃO JFF. Métodos de avaliação da funcionalidade do idoso e sua correlação com a CIF: revisão integrativa. Trabalho de conclusão de curso (Especialização em Saúde da Pessoa Idosa) - Universidade de Brasília, Campus Darcy Ribeiro, 2017.

35. ROSA AMP, et al. Propriedades métricas do Timed Up and Go Test no idoso: revisão integrativa da literatura. Revista Investigação em Enfermagem, 2017; 21-31.

36. SCHERRER G, et al. Fatores associados à dependência de idosos residentes em instituições públicas. Remecs. 2019; 4(6): 3-11.

37. SILVA CS, et al. Capacidade funcional de idosos em instituição de longa permanência. Id on Line Rev. Mult. Psic, $2017 ; 11(38)$.

38. SOUSA HJS, et al. Análise da abordagem fisioterapêutica nas alterações senescentes e senis do sistema musculoesquelético. Revista da FAESF, 2019; 3(2).

39. SOUZA LHR, et al. Capacidade funcional de idosos residentes em uma instituição de longa permanência. Acta Biomedica Brasiliensia. 2017; 8(2).

40. TALARSKA D, et al. Is independence of older adults safe considering the risk of falls?. BMC Geriatrics, 2017; 17(66). 